\title{
Fenómeno de Lúcio: Resposta ao Tratamento Alternativo com Poliquimioterapia Multibacilar
}

\author{
Camila Araujo Dantas', Caroline Medeiros Prohmann', Lorena Barcelos e Silva', Rafael Figueiredo Gatti ', Thalita Marçal

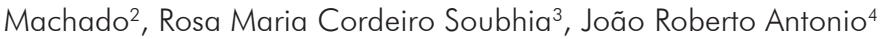 \\ 'Residente em Dermatologia/Resident of Dermatology, Faculdade Estadual de Medicina de São José do Rio Preto (FAMERP), São \\ José do Rio Preto, Brasil \\ ${ }^{2}$ Dermatologista/Dermatologist pela Faculdade Estadual de \\ Medicina de São José do Rio Preto (FAMERP), membro da Sociedade Brasileira de Dermatologia (SBD) \\ ${ }^{3}$ Professora e chefe do Ambulatório de Hanseníase do serviço de Dermatologia do Hospital de Base de São José do Rio Preto - \\ FAMERP, Brasil \\ ${ }^{4}$ Dermatologista/Dermatologist, Membro da SBD e Chefe do Ambulatório de Dermatologia e Professor Emérito da Faculdade \\ Estadual de Medicina de São José do Rio Preto (FAMERP), São José do Rio Preto, Brasil
}

RESUMO - A hanseníase é uma doença infectocontagiosa de evolução crónica, causada pelo Mycobacterium leprae. Seu curso crónico pode ser interrompido por episódios reacionais agudos ou subagudos. O fenómeno de Lúcio é uma reação imunológica vasculítica que ocorre em doentes com lepra lepromatosa. Surge com máculas purpúricas que evoluem com necrose e ulceração, de forma ascendente, deixando cicatriz atrófica. Os autores descrevem o caso de um paciente de 73 anos, sexo masculino, com lesões ulceronecróticas em membros inferiores e exposição tendinosa em calcâneo. Com base em dados clínico-laboratoriais e exame dermatológico, confirmado diagnóstico de lepra lepromatosa com vasculite do tipo fenómeno de Lúcio. Evoluiu com boa resposta ao esquema alternativo de tratamento com poliquimioterapia multibacilar.

PALAVRAS-CHAVE - Lepra Lepromatosa; Mycobacterium leprae; Quimioterapia Combinada.

\section{Lucio's Phenomenon: Response to Alternative Treatment with Multibacilar Polychemotherapy}

ABSTRACT - Leprosy is an infectious disease of chronic evolution caused by Mycobacterium leprae. Its chronic course can be interrupted by acute or subacute reactional episodes. Lucius phenomenon is a vasculitic immune reaction that occurs in patients with lepromatous leprosy. It appears with purpuric macules that progress to ascending necrosis and ulceration and heal with an atrophic scar. The authors describe the case of a 73-year-old male patient with ulceronecrotic lesions in the lower limbs and tendinous calcaneal exposure. Clinical-laboratory data and dermatological examination confirmed the diagnosis of lepromatous leprosy with vasculitis of the Lucius-like type. It progressed with a good response to the alternative treatment with multibacillary multidrug therapy. KEYWORDS - Drug Therapy, Combination; Leprosy, Lepromatous; Mycobacterium leprae.

\section{INTRODUÇÃO}

A hanseníase é uma doença infectocontagiosa de evolução crónica, causada pelo Mycobacterium leprae - um bacilo álcool-ácido resistente de alta infecciosidade e baixa patogenicidade, descrito pelo médico norueguês Gerard H. Armauer Hansen em 1873.' Atualmente é aceite que a sua transmissão ocorre através do contato com indivíduos doentes, principalmente por microorganismos eliminados pelas mucosas nasal e oral. De acordo com dados divulgados recentemente pela Organização Mundial de Saúde (OMS), Índia, Brasil e Indonésia notificam mais de 10000 novos pacientes anualmente, representando $81 \%$ dos pacientes recém- diagnosticados e notificados no mundo. Países com poucos casos, incluindo países Europeus, devem manter serviços de
Correspondência: Camila Araujo Dantas

Rua Abdo Muanis, 1001 Bloco 1 Apto 93

São José do Rio Preto - São Paulo. CEP: 15090-140

E-mail: milladantas@hotmail.com

DOI: https://dx.doi.org/10.29021/spdv.76.2.804
Recebido/Received

14 Setembro/September 2017

Aceite/Accepted

09 Janeiro/January 2018 


\section{Caso Clínico}

vigilância e referência, sobretudo devido à migração de países onde a hanseníase é endêmica. ${ }^{2}$

A doença é caracterizada por manifestações da pele e do sistema nervoso periférico, mas outros órgãos também podem ser afetados, como o sistema reticuloendotelial, testículos e medula óssea. De acordo com a classificação de Madri, são quatro as formas clínicas: indeterminada, tuberculóide, lepromatosa e borderline. ${ }^{3}$ Recentemente foi descrita outra espécie de micobactéria (agente FJ924 ou Mycobacterium lepromatosis), geneticamente distinta do Mycobacterium leprae como agente causal da hanseníase lepromatosa difusa em indivíduos procedentes do México que desenvolveram fenómeno de Lúcio fatal. Esta observação não foi confirmada de forma independente em outros estudos e o significado clínico (em magnitude e distribuição) do M. lepromatosis como agente causador da hanseníase lepromatosa difusa (hanseníase de Lúcio) permanece incerto e até mesmo questionado. ${ }^{4}$

A evolução para cura ou para cada espectro clínico da doença depende do estado imunológico do paciente, avaliado pela reação cutânea de Mitsuda. Esta constitui um parâmetro imunológico para avaliar a resposta tardia a uma sequência de eventos que se seguem à fagocitose dos bacilos contidos na suspensão, pelos histiócitos. Através deste, é possível avaliar a resposta imune celular nos indivíduos, resposta esta intimamente relacionada com a progressão e manifestação clínica da doença. ${ }^{5}$

O curso crónico da hanseníase pode ser interrompido por alterações agudas no balanço imunológico entre hospedeiro e o bacilo, denominadas "reações ou estados reacionais". As reações do tipo 1 são mediadas por imunidade celular e podem ser de melhoria - reação reversa ou up grading reaction, que ocorre nos pacientes que iniciaram tratamento - ou de agravamento - down grading reaction, ocorrendo nos doentes virgens de tratamento. As reações do tipo 2 são mediadas por imunocomplexos e produção de citocinas e ocorrem nas formas lepromatosa e borderline. $\bigcirc$ fenómeno de Lúcio é considerado uma variante da reação tipo 2,5 mas alguns autores preferem classificá-lo como um terceiro tipo de reação, caracterizada por necrose de arteríolas. ${ }^{3,5,7}$

O fenómeno de Lúcio ou eritema necrosante é uma manifestação reacional grave relativamente rara no Brasil, comparado com os casos relatados no México e América Central, ${ }^{7-11}$ e que ocorre nos portadores de hanseníase lepromatosa forma difusa. ${ }^{7,8,11}$ É uma vasculite aguda, caracterizada por necrose das arteríolas e endotélio altamente parasitado por bacilos íntegros. Clinicamente, apresenta-se com lesões purpúricas que evoluem com necrose e ulceração. ${ }^{12-16}$ Afetam geralmente os membros inferiores de forma ascendente, menos comumente os membros superiores e glúteos, raramente a face, e evolvem com cicatrização atrófica. ${ }^{7} \bigcirc$ tratamento é específico para hanseníase, ${ }^{17}$ embora alguns autores defendam o uso concomitante de corticoide sistêmico/talidomida por se tratar de uma forma reacional. ${ }^{5}$

\section{CASO CLÍNICO}

Doente do sexo masculino, 73 anos, caucasiano, casado, aposentado, natural de Bálsamo, procedente de São José do Rio Preto. Relatava desde há um ano perda de 10 kg e "feridas nas pernas", com eliminação de secreção de odor fétido, sanguinolenta. De antecedentes pessoais, referiu tabagismo por 30 anos e negava etilismo. À admissão, encontrava-se em regular estado geral, com sinais vitais estáveis e apirético. Ao exame dermatológico tinha fácies infiltrada com madarose distal de sobrancelhas, perda de pelos em lesões cutâneas, obstrução nasal e hipoestesia em pernas e lesões ulceradas e necróticas em dorso dos pés (Fig. 1A) e membros inferiores (Fig. 1B), com exposição do tendão calcâneo à direita (Fig. 2A). A baciloscopia da pele demonstrou bacilos álcool-acido-resistentes (BAAR) com formação de globias, índice baciloscópico $=1$, com $4 \%$ de bacilos íntegros e a biópsia evidenciou vasculite leucocitoclástica com necrose fibrinóide (Fig.s $3 \mathrm{~A}$ e 3B). Os exames complementares não revelaram alterações do complemento sérico, nem anticorpos anti-nucleares e as sorologias para hepatites foram negativas. Estes aspectos clínicos, laboratoriais e histopatológicos conduziram ao diagnóstico de
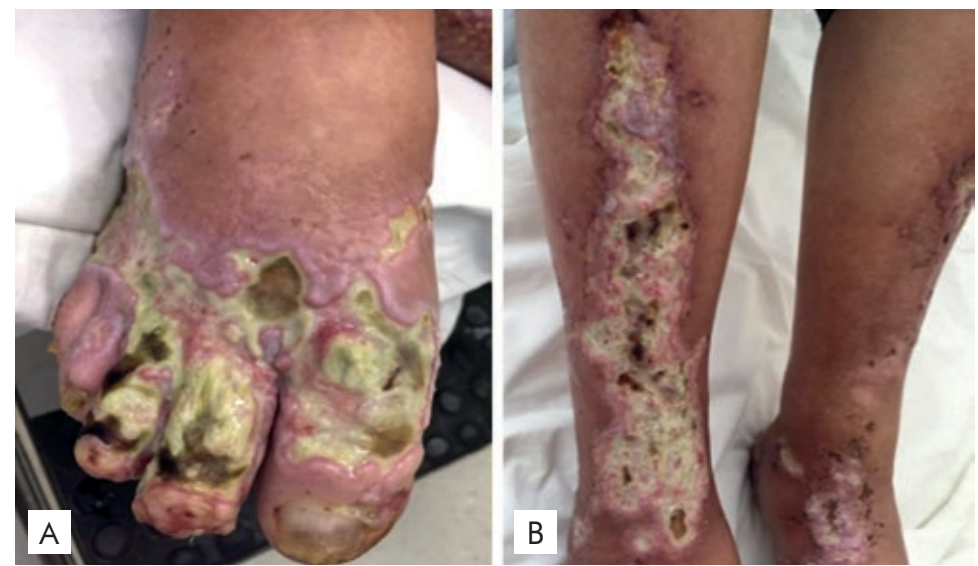

Figura 1 - Ulcerações necróticas em dorso dos pés (A) e face anterior da perna (B). 


\section{Caso Clínico}
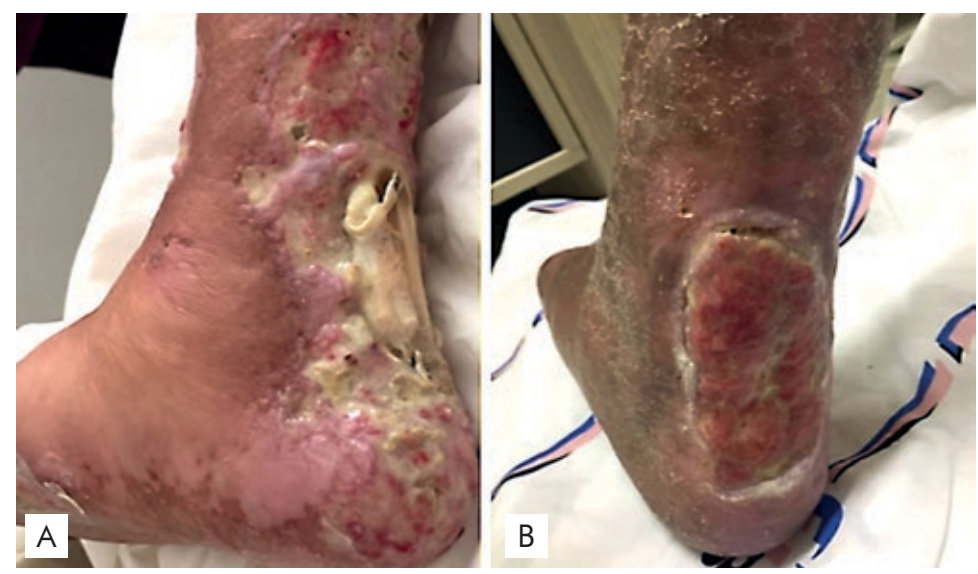

Figura 2 - Exposição do tendão calcâneo à direita (A) e 5 meses depois (B)

hanseníase lepromatosa na sua forma difusa e fenómeno de Lucio. Iniciou esquema de poliquimioterapia multibacilar preconizado pela OMS para forma multibacilar com rifampicina, clofazimina e dapsona, associado a prednisona $60 \mathrm{mg} /$ dia. Quinze dias depois evoluiu com plaquetopenia 98,000 $\mu \mathrm{L}$, anemia hemolítica (queda da hemoglobina de $10,2 \mathrm{~g} / \mathrm{dL}$ para $7,4 \mathrm{~g} / \mathrm{dL}$ ) e dosagem de glicose 6 fosfato desidrogenase (G6PD) de 1,9 U/g Hb (valor de referência: superior a 2,2 U/g $\mathrm{Hb}$ ), com necessidade de hemotransfusão. A biópsia de medula óssea evidenciou hipercelularidade à custa de histiócitos, contendo numerosos BAAR, compatível com comprometimento medular por hanseníase. $O$ esquema clássico foi substituído pelo alternativo, indicado para os casos intolerantes à dapsona e à rifampicina (clofazimina dose mensal de $300 \mathrm{mg}$ supervisionada e dose diária de $50 \mathrm{mg}$, autoadministrada + ofloxacina na dose mensal de $400 \mathrm{mg}$ supervisionada e dose diária de $400 \mathrm{mg}$, autoadministrada + minociclina na dose mensal de $100 \mathrm{mg}$ supervisionada e dose diária de $100 \mathrm{mg}$ autoadministrada), sendo mantida a corticoterapia.

Apesar da proposta de amputação do membro inferior direito pela ortopedia, foi mantido o tratamento médico e acionadas sessões de câmera hiperbárica com melhora clínica e cicatrização satisfatória das lesões ulceradas e da exposição tendinosa após 5 meses (Fig. 2B), e com preservação do membro acometido.

\section{DISCUSSÃO}

Neste paciente perante as ulcerações necróticas, infiltração difusa da pele, madarose, obstrução nasal e alterações sensoriais, e pelos achados histopatológicos foi feito o diagnóstico de hanseníase lepromatosa forma difusa e fenómeno de Lúcio. Histopatologicamente, este caracteriza-se por uma proliferação exacerbada dos bacilos de Hansen, que invadem a parede dos vasos sanguíneos e agridem as células endoteliais, causando proliferação endotelial e diminuição do lúmen vascular, fato este que, associado a reações inflamatórias e a alterações no sistema da coagulação, causa trombose vascular, isquemia, infarto e necrose tecidual, gerando as alterações histopatológicas características deste fenómeno. Os 3 critérios que confirmam o fenómeno de Lúcio (lesões cutâneas ulceradas e úlcero-necróticas, trombose vascular e proliferação de bacilos de Hansen íntegros no endotélio de arteríolas), estavam presentes no caso relatado.

O diagnóstico diferencial do fenómeno de Lúcio inclui o eritema nodoso lepromatoso necrótico (ENL necroticans), duas variantes da reação hansênica do tipo 2 e deve considerar a vasculite leucocitoclástica como entidade à parte, independente de hanseníase. ${ }^{5}$ O fenómeno de Lúcio está mais associado aos pacientes não diagnosticados, ainda sem tratamento, ocorre na evolução da hanseníase de Lúcio e é caracterizado por aparecimento de máculas eritematosas dolorosas, com
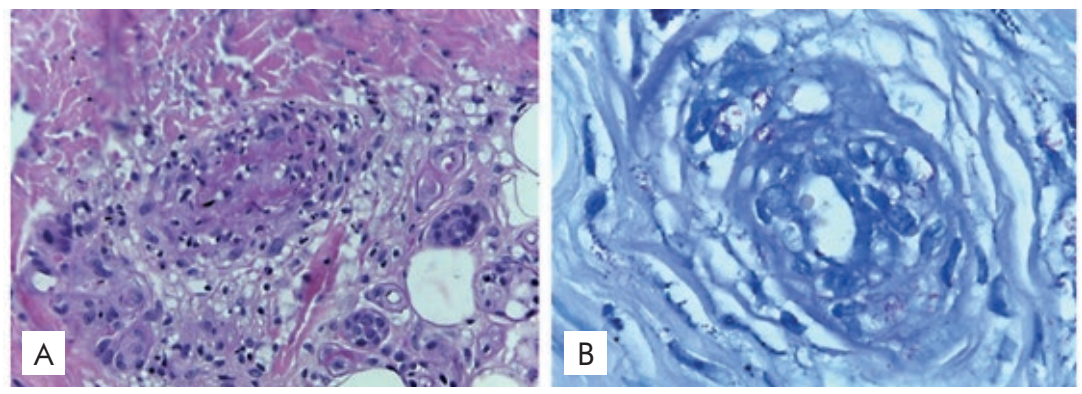

Figura 3 - Exame Anatomopatológico cutâneo: (A) Vaso sanguíneo exibindo lesão de célula endotelial, depósito de fibrina no interior da luz e neutrófilos na parede (HE-400x). (B) Presença de BAAR nas células endoteliais. 


\section{Caso Clínico}

evolução para necrose central e posterior ulceração, mais comuns nas extremidades, poupando face, com pouca sintomatologia sistêmica. Distingue-se do eritema nodoso leprótico que é observado mais frequentemente na forma difusa da hanseníase lepromatosa e que se caracteriza por necrose em lesões de eritema nodoso. Localiza-se mais frequentemente na face e membros, sendo geralmente acompanhado de sinais e sintomas sistêmicos, com poucos bacilos, sendo a ulceração um evento raro.

Neste paciente, foi instituído tratamento com poliquimioterapia multibacilar, porém após 2 semanas o paciente evoluiu com anemia importante e plaquetopenia, necessitando de hemotransfusão. $\bigcirc$ esquema terapêutico foi então substituído por poliquimioterapia alternativa, que demonstrou a sua eficácia mesmo em quadros avançados com exposição tendinosa, como do paciente em questão. Como adjuvante, foi associado tratamento com sessões de câmera hiperbárica, já documentado na literatura como acelerador do processo de cicatrização de feridas resistentes. ${ }^{18}$

O relato acima demonstra aspectos rotineiros na evolução de pacientes com hanseníase, em que o retardo no diagnóstico pode levar a um quadro grave ou sequelas permanentes, em muitos casos por falta de conhecimento sobre a doença pela população. Por isso o reforço do papel fundamental do dermatologista no reconhecimento de sinais iniciais da doença, dado que muitas vezes é o primeiro profissional médico a ser procurado pelo paciente, e a pronta instituição do tratamento permite menos complicações e melhor controle do ciclo de transmissão da doença.

Conflitos de interesse: Os autores declaram não possuir conflitos de interesse.

Suporte financeiro: $O$ presente trabalho não foi suportado por nenhum subsídio ou bolsa.

Confidencialidade dos dados: Os autores declaram ter seguido os protocolos do seu centro de trabalho acerca da publicação dos dados de doentes.

Protecção de pessoas e animais: Os autores declaram que os procedimentos seguidos estavam de acordo com os regulamentos estabelecidos pelos responsáveis da Comissão de Investigação Clínica e Ética e de acordo com a Declaração de Helsínquia da Associação Médica Mundial.

Conflicts of interest: The authors have no conflicts of interest to declare.

Financing Support: This work has not received any contribution, grant or scholarship.

Confidentiality of data: The authors declare that they have followed the protocols of their work center on the publication of data from patients.

Protection of human and animal subjects: The authors declare that the procedures followed were in accordance with the regulations of the relevant clinical research ethics committee and with those of the Code of Ethics of the World Medical Association (Declaration of Helsinki).

\section{REFERÊNCIAS}

1. Karg E, Korom I, Varga E, Ban G, Turi S. Congenital 1. Belda Júnior W, Di Chiacchio N, Criado PR. Tratado de Dermatologia. $2^{a}$ ed. São Paulo: Atheneu; 2014.

2. World Health Organization. Global Leprosy Strategy: Accelerating towards a leprosy-free world. Geneve: WHO; 2016.

3. Souza CS. Hanseníase: formas clínicas e diagnóstico diferencial. Med Ribeirão Preto. 1997;30:325-34.

4. Vera-Cabrera L, Escalante-Fuentes WG, Gomez-Flores M, Ocampo-Candiani J, Busso P, Singh P, et al. Case of diffuse lepromatous leprosy associated with "Mycobacterium lepromatosis". J Clin Microbiol. 201;49:4366-8.

5. Azulay-Abulafia L. Revendo a hanseníase de Lucio e o fenómeno de Lucio. Med Cutan Iber Lat Am 2005;33:12533.

6. Rivitti, Evandro A. Manual de dermatologia clínica de Sampaio e Rivitti. São Paulo: Artes Médicas; 2014.

7. Helmer KA, Fleischfresser I, Kucharski-Esmanhoto LD, Neto JF, Santamaria JR. The Lucio's phenomenon (necrotizing erythema) in pregnancy. An Bras Dermatol. 2004;79:205-10.

8. Antonio JR, Cipullo JP, Soubhia RC, D'Ávila SC, Neto DS, Pereira AS. Lucio's phenomenon. RBM. 2003;62:103-6.

9. Ribeiro SLE, Erilane, Pereira HLA, Sales LS. Vasculite na hanseníase mimetizando doenças reumáticas. Rev Bras Reumatol. 2007; 47:140-4.

10. Bernadat JP, Faucher JF, Huerre M. Diffuse lepromatous leprosy disclosed by cutaneous vasculitis: the Lucio phenomenon. Ann Dermatol Venereol. 1996;123:21-3.

11. Report of the International Leprosy Association, Technical Forum Paris, France 22-28 February 2002. Int J Leprosy. 2002;70:21-2.

12. Azulay-Abulafia, L., and L. Spinelli. Revendo a hanseníase de Lúcio e o Fenômeno de Lúcio. Med Cutan Iber Lat Am. 2005;33: 125-33.

13. Peixoto AB, Portela OS, Leal FR, Brotas AM, Rodrigues NS. Lucio's Phenomenon. Case study of an exceptional response to treatment exclusively with multibacillary multidrug therapy. An Bras Dermatol. 2013;88:93-6.

14. Soares R, Silva RC, Ceribelli AC, Ura . Lucio's phenomenon - Case report. Hansenologia Internationalis. 2009; 34:47-51.

15. Monteiro R, Tiezzi MG, Abreu MA, Oliveira CC, Roncada EV, Ortigosa LC. Lucio's phenomenon: another case reported in Brazil. An Bras Dermatol. 2012; 87:296-300.

16. Pereira AC Jr. Hanseníase de Lúcio. An Bras Dermatol. 1993;68:33-40.

17. Ferreira LM, Medeiros AP, Gasparelo LG. Fenómeno de Lúcio. Rev Soc Port Dermatol Venereol. 2015;73: 485-8.

18. Gillis TP, Scollard DM, Lockwood DN. What is the evidence that the putative Mycobacterium lepromatosis species causes diffuse lepromatous leprosy? Lepr Rev. 2011;82:205-9. 